\title{
Connection between Genetic and Clinical Data in Bipolar Disorder
}

\author{
Erling Mellerup ${ }^{1 *}$, Ole Andreassen ${ }^{3}$, Bente Bennike ${ }^{1}$, Henrik Dam ${ }^{2}$, Srdjan Durovic ${ }^{4}$, Thomas Hansen ${ }^{5}$, \\ Ingrid Melle ${ }^{3}$, Gert Lykke Møller ${ }^{7}$, Ole Mors ${ }^{6}$, Pernille Koefoed ${ }^{1,2}$
}

1 Laboratory of Neuropsychiatry, Department of Neuroscience and Pharmacology, University of Copenhagen, Blegdamsvej, Copenhagen, Denmark, 2 Psychiatric Centre Copenhagen, Department O, Copenhagen University Hospital, Rigshospitalet, Blegdamsvej, Copenhagen, Denmark, 3 Department of Psychiatry, Oslo University Hospital and Institute of Psychiatry, University of Oslo, Kirkeveien, Oslo, Norway, 4 Department of Medical Genetics, Oslo University Hospital and Institute of Psychiatry, University of Oslo, Kirkeveien, Oslo, Norway, 5 Department of Biological Psychiatry, Mental Health Centre Sct. Hans, Copenhagen University Hospital, Boserupvej 2, Roskilde, Denmark, 6 Centre for Psychiatric Research, Aarhus University Hospital, Skovagervej, Risskov, Denmark, 7 Genokey ApS, ScionDTU, Technical University Denmark, Agern Allé, Hoersholm, Denmark

\begin{abstract}
Complex diseases may be associated with combinations of changes in DNA, where the single change has little impact alone. In a previous study of patients with bipolar disorder and controls combinations of SNP genotypes were analyzed, and four large clusters of combinations were found to be significantly associated with bipolar disorder. It has now been found that these clusters may be connected to clinical data.
\end{abstract}

Citation: Mellerup E, Andreassen O, Bennike B, Dam H, Durovic S, et al. (2012) Connection between Genetic and Clinical Data in Bipolar Disorder. PLoS ONE 7(9): e44623. doi:10.1371/journal.pone.0044623

Editor: Chunyu Liu, University of Illinois at Chicago, United States of America

Received March 26, 2012; Accepted August 6, 2012; Published September 20, 2012

Copyright: (c) 2012 Mellerup et al. This is an open-access article distributed under the terms of the Creative Commons Attribution License, which permits unrestricted use, distribution, and reproduction in any medium, provided the original author and source are credited.

Funding: No external funding have supported this study.

Competing Interests: The authors have declared that no competing interests exist.

*E-mail: mellerup@sund.ku.dk

\section{Introduction}

Modern analytical methods, particularly in the field of molecular genetics, produce large amounts of data that pose a challenge to statistical and data-mining methods for extracting useful information [1,2]. Thus, in polygenic diseases finding disease related genetic changes, among the vast number of changes (e.g., millions of SNPs), is a daunting task. In a recent study [3], 803 SNPs in samples from 607 bipolar patients and 1355 control subjects were analyzed. All the SNPs were from genes selected based on theoretical and experimental studies that suggested that signal transduction and particular ion channels were involved in bipolar disorder [4-6]. The number of combinations of 3 SNP genotypes was counted in the material [3]. The theoretical number of combinations of 3 SNP genotypes taken from 803 SNPs is $2,321,319,627\left(803 ! / 3 !(803-3) ! \times 3^{3)}\right.$, and as many as $1,985,613,130$ combinations were found in the participants. $1,719,002,329$ combinations were common between controls and patients, 208,699,590 combinations were found in controls only, and 57,911,211 combinations were found in patients only, of these 45,285,770 occurred only once, and not more than 1181 combinations were shared by 9 or more patients. None of the 803 single SNPs or the nearly two billion of SNP genotype combinations showed a statistically significant association with bipolar disorder. However, among the 1181 combinations shared by 9 or more patients and no controls, four clusters of combinations, were identified that were significantly associated with bipolar disorder. Within a cluster, each patient had a personal pattern of SNP genotypes that was somewhat similar to the patterns of other patients in the same cluster, but quite different from the patterns of patients in the other three clusters, hereby suggesting an extreme degree of genetic heterogeneity $[3,7]$.

\section{Results}

The four clusters, are shown in Table 1, 2, 3, 4. Of the 607 patients, 156 were members of the 4 clusters. The clusters contained 41, 48, 41, and 37 patients; 11 patients were members of two clusters, and no patient was a member of three clusters. The clusters contained 60, 60, 65, and 53 SNP genotypes; 29 SNP genotypes were located in two clusters, and one SNP genotype (rs1380452 positioned in the ANK3 gene) was located in three clusters.

The 156 patients were subdivided into the 4 clusters and into three groups based on three geographic areas in Scandinavia (Oslo, Aarhus, and Copenhagen). The available clinical data were not the same in the three areas. The number of hypomanic, manic and depressive episodes was available from the Norwegian patients, the number of hospital admissions and the presence of alcohol dependence was available from the patients from Copenhagen (Tables 5, 6, 7).

Table 5 shows number of hypomanic and manic episodes and depressive episodes for the single patients. The median for the number of hypomanic and manic episodes is 3 , and the median for the number of depressive episodes is 3.5. Patients having numbers of episodes above the median for both hypomanic and manic episodes and depressive episodes (more serious disease) were compared with patients having at least one type of episodes below or equal to the medians $(\mathrm{p}=0.0045$ for clusters $1+2+4$ versus cluster 3). 
Table 1. Cluster 1 . defined by SNP1 $=$ AVPR1B_rs33976516 $=1$.

\begin{tabular}{|c|c|c|c|}
\hline SNP2 & SNP3 & $\mathbf{G T}^{\mathbf{a}}$ & Patients $^{\mathbf{b}}$ \\
\hline ANK3_rs2288358 & SCN2B_rs8192614 & 20 & 94126132166333393409413528 \\
\hline ANK3_rs2288358 & CAMKK2_rs11065502 & 20 & 94126132166333393409413528 \\
\hline ANK3_rs2288358 & PPP2R2C_rs17721365 & 20 & 94126132166333393409413528 \\
\hline ANK3_rs2288358 & ANK3_rs10994322 & 20 & 94126132166333393409413528 \\
\hline ANK3_rs2288358 & KCNC2_rs1880840 & 20 & 94126132166333393409413528 \\
\hline ANK3_rs4948255 & KCNQ2_rs3787119 & 20 & 94126151166333393409413528 \\
\hline ANK3_rs4948255 & KCNN3_rs12029542 & 20 & 94126151166333393409413528 \\
\hline ANK3_rs4948255 & ATP1A3_rs4803520 & 20 & $94126132151 \quad 166333393409528$ \\
\hline ANK3_rs4948255 & ATP1A3_rs2217342 & 20 & 94126132151166333393409528 \\
\hline ANK3_rs4948255 & CACNG2_rs4821512 & 21 & 94132151166333393409413528 \\
\hline CNTNAP2_rs6945513 & NFASC_rs17415523 & 00 & 105149197200210231278333390 \\
\hline CNTNAP2_rs6945513 & ANK3_rs17805456 & 00 & 6105149197200210231333390 \\
\hline CNTNAP2_rs6945513 & ANK3_rs7895653 & 00 & 6105149200210231278333390 \\
\hline CNTNAP2_rs6945513 & ANK3_rs10994322 & 00 & 6105149200210231278333390 \\
\hline CNTNAP2_rs6945513 & KCNN3_rs951241 & 00 & 6105149200210231278333390 \\
\hline KCNN3_rs6699080 & SCN2B_rs8192614 & 20 & 694100126151278285366409593 \\
\hline KCNN3_rs6699080 & TNR_rs2236885 & 20 & 694100126151278285366409593 \\
\hline KCNN3_rs6699080 & ANK3_rs4359155 & 20 & 694100126151278285409593 \\
\hline KCNN3_rs6699080 & MCTP2_rs3784644 & 20 & 694100151278285366409593 \\
\hline P2RX7_rs6489794 & OLIG2_rs762178 & 11 & 42151166210248330393421511 \\
\hline P2RX7_rs6489794 & CACNG2_rs2284016 & 10 & 57151248330356366393421511 \\
\hline P2RX7_rs6489794 & ANK3_rs2393602 & 10 & 57210231248304330366421511 \\
\hline KCNN3_rs1218575 & CREB1_rs2551921 & 11 & 57126151200210231393436511593 \\
\hline KCNN3_rs1218575 & CNTNAP2_rs2620460 & 11 & 126151200231248393409436545 \\
\hline KCNN3_rs1218575 & ANK3_rs1010556 & 12 & 65794200393409511545593 \\
\hline ANK3_rs10821695 & TRPM2_rs1556314 & 01 & 4294114231285333393421575 \\
\hline ANK3_rs10821695 & TRPM2_rs734336 & 01 & 4294114166231285333393575 \\
\hline NFASC_rs16854930 & IMPA2_rs628419 & 11 & 5194132248390393394511575593 \\
\hline NFASC_rs16854930 & SCN1B_rs8100085 & 11 & 166285356393409413436511575 \\
\hline NCAM1_rs12794326 & TNR_rs2239821 & 21 & 88149200210304330390394528596 \\
\hline NCAM1_rs12794326 & CNTN1_rs7315781 & 20 & 149200210330390393528575596 \\
\hline CNTN2_rs16855045 & P2RX7_rs1718161 & 11 & 51149166210231248330393413421593 \\
\hline CNTN2_rs16855045 & CACNG2_rs2283970 & 10 & 5157149210248330333356413421 \\
\hline ANK3_rs1010556 & ANK3_rs10994195 & 01 & 425188114149153166278366413421 \\
\hline ANK3_rs1010556 & CNTNAP2_rs1024676 & 20 & 65794132304409511545593 \\
\hline KCNN3_rs6426998 & TNR_rs1385541 & 20 & 694126278285393409421593 \\
\hline KCNQ2_rs6122454 & NRCAM_rs759548 & 01 & 57149200285304356421511545 \\
\hline SCN4B_rs868344 & ANK3_rs7893313 & 21 & 35428894100149333356409 \\
\hline ANK3_rs1380452 & ANK3_rs10994171 & 20 & 657100105132304409436511593 \\
\hline SCN4B_rs678262 & ANK3_rs2018783 & 02 & 4294100114248304393409436 \\
\hline CACNG2_rs2283970 & CNTN2_rs3767298 & 01 & 5157149210248333356413421 \\
\hline P2RX7_rs1718161 & CNTN2_rs3767298 & 11 & 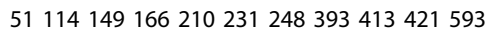 \\
\hline ATP1A3_rs4803520 & KCNN3_rs6426998 & 02 & 694126151285366393409421 \\
\hline ANK3_rs17805456 & CNTNAP2_rs10808044 & 02 & 6105149153197210231366390413 \\
\hline NCAM1_rs584427 & AQP4_rs3763043 & 20 & 35151200366390409413421436545 \\
\hline NFASC_rs2802853 & KCNQ3_rs869710 & 10 & 578894105248285356393575593 \\
\hline
\end{tabular}

Cluster 1 contains 41 patients, 46 combinations of 3 SNP genotypes, and 58 SNP genotypes.

a) GT = genotype for SNP2 and SNP3 (0: Normal homozygote.1: Heterozygote. 2: Variant homozygote); b) Dummy ID. doi:10.1371/journal.pone.0044623.t001 
Table 2. Cluster 2. defined by SNP1 $=$ KCNN3_rs884664 $=2$.

\begin{tabular}{|c|c|c|c|}
\hline SNP2 & SNP3 & $\mathbf{G T}^{\mathbf{a}}$ & Patients $^{\mathbf{b}}$ \\
\hline ANK3_rs2018783 & KCNQ3_rs7002144 & 00 & 2224153212359375553573584585 \\
\hline ANK3_rs2018783 & KCNQ3_rs10217015 & 00 & 2224153212375553573584585 \\
\hline ANK3_rs2018783 & CNTNAP2_rs1587048 & 00 & 2224305351359421500553573 \\
\hline ANK3_rs2018783 & CNTNAP2_rs1524339 & 01 & 22153212351359421573584585 \\
\hline ANK3_rs2018783 & CNTN1_rs11178111 & 00 & 2224153305351359573584585 \\
\hline CAMKK2_rs2686343 & NCAM1_rs584427 & 00 & 2250154188201351359500524 \\
\hline CAMKK2_rs2686343 & SCN2A_rs17184707 & 01 & 2291154188201351383417422 \\
\hline CAMKK2_rs2686343 & NFASC_rs10900430 & 01 & 225091188201212280359422 \\
\hline CAMKK2_rs2686343 & NFASC_rs11240304 & 01 & 2291154188201351383417422 \\
\hline CAMKK2_rs2686343 & NFASC_rs7535216 & 01 & 2291154188201351383417422 \\
\hline CNTNAP2_rs10808044 & BACE1_rs522843 & 00 & 5247891156178293375421422503553 \\
\hline CNTNAP2_rs10808044 & BACE1_rs473210 & 00 & 5247891156178293375421422553 \\
\hline CNTNAP2_rs10808044 & BACE1_rs525493 & 00 & 5247891156178293421422553 \\
\hline CNTNAP2_rs10808044 & BACE1_rs477036 & 00 & 5247891156178293421422553 \\
\hline BDNF_rs908867 & AQP4_rs3875089 & 10 & 52450123156176201359378500 \\
\hline BDNF_rs908867 & NRG1_rs2975500 & 10 & 52450123156176201378500 \\
\hline BDNF_rs908867 & KCNQ3_rs17575754 & 10 & 52450123156176359378500 \\
\hline IMPA2_rs636173 & KCNC1_rs7110441 & 20 & 386274154201422500524526553 \\
\hline IMPA2_rs636173 & KCNC1_rs16934680 & 20 & 386274154201422500524526553 \\
\hline CNTNAP2_rs9640245 & TNR_rs859437 & 12 & 2774176189283383500526585 \\
\hline CNTNAP2_rs9640245 & TNR_rs12119177 & 10 & 2774176189283383500526585 \\
\hline ANK3_rs16914644 & KCNN3_rs11264248 & 12 & 386274123147178201305422 \\
\hline ANK3_rs16914644 & KCNN3_rs6695232 & 10 & 386274123147178201305422 \\
\hline CACNG2_rs2284010 & KCNQ3_rs713148 & 02 & 627886147156176189212359422503526 \\
\hline CACNG2_rs2284010 & KCNQ3_rs17595945 & 02 & 627886156176189212359422526 \\
\hline KCNC3_rs1559133 & PDE4B_rs4288570 & 10 & 2262147212280375422500538 \\
\hline KCNC3_rs1559133 & ANK3_rs10821695 & 10 & 62147212280293375422526538 \\
\hline KCNC2_rs2926150 & CNTN2_rs11240351 & 11 & 22387886123176189378503 \\
\hline KCNC2_rs2926150 & BACE1_rs522843 & 10 & 54778123156176378417503 \\
\hline NRCAM_rs11767318 & BACE1_rs525493 & 10 & 56278178293305421422585 \\
\hline NRCAM_rs11767318 & BACE1_rs477036 & 10 & 56278178293305421422585 \\
\hline CAMKK2_rs11065502 & SCN2A_rs17184707 & 11 & 2791176188201212305359375422 \\
\hline CAMKK2_rs11065502 & KCNN3_rs11264254 & 11 & 2730156176201305417422524 \\
\hline KCNQ3_rs713148 & KCNC2_rs1379963 & 21 & 47627886156189421422503585 \\
\hline CNTN1_rs11178111 & NRCAM_rs10953566 & 10 & 2747153176178212359524584585 \\
\hline MBP_rs12962017 & PPP2R2C_rs4689408 & 11 & 53062135154351378500538 \\
\hline KCNN3_rs11264254 & P2RX7_rs1718134 & 21 & 5387491188212314421584 \\
\hline KCNA1_rs1048500 & P2RX7_rs503720 & 00 & 22475062135293359417526 \\
\hline KCNA2_rs3887820 & KCNQ2_rs6062929 & 11 & 2430147153154156188305503 \\
\hline IMPA2_rs3859296 & PPP2R2C_rs16838658 & 11 & 223847176283417524526584 \\
\hline NFASC_rs11240304 & KCNQ2_rs6090403 & 11 & 2291188189229293383417422 \\
\hline BDNF_rs11030102 & SLC12A6_rs17236791 & 11 & 304774188280293375422538573 \\
\hline NFASC_rs10900430 & KCNQ2_rs6090403 & 11 & $2291 \quad 188189229293383417422$ \\
\hline SCN1B_rs8100085 & YWHAH_rs929036 & 02 & 22247486153154383417538 \\
\hline SPTBN4_rs4803342 & MAP2_rs17745941 & 11 & 30506278283422500524553 \\
\hline
\end{tabular}

Cluster 2 contains 48 patients, 45 combinations of 3 SNP genotypes, and 60 SNP genotypes.

a) GT = genotype for SNP2 and SNP3 (0: Normal homozygote.1: Heterozygote. 2: Variant homozygote); b) Dummy ID.

doi:10.1371/journal.pone.0044623.t002 
Table 3. Cluster 3.defined by SNP1 $=$ CACNG2_rs2179871 $=2$.

\begin{tabular}{|c|c|c|c|}
\hline SNP2 & SNP3 & $\mathbf{G T}^{\mathbf{a}}$ & Patients $^{\mathbf{b}}$ \\
\hline KCNN3_rs6426998 & NCAM1_rs7130671 & 21 & 4465119146224227232290380417599602 \\
\hline KCNN3_rs6426998 & KCNC2_rs1458606 & 21 & 4465119224290331380417515599602 \\
\hline KCNN3_rs6426998 & CAMKK2_rs1653594 & 21 & 44119146224227290331380417599602 \\
\hline KCNN3_rs6426998 & CAMKK2_rs1140886 & 21 & 4465119290331380417515599602 \\
\hline KCNN3_rs6426998 & CAMKK2_rs1063843 & 21 & 4465119290331380417515599602 \\
\hline KCNN3_rs6426998 & SPTBN4_rs17656504 & 20 & 4465146224227232290380515602 \\
\hline KCNN3_rs6426998 & CNTNAP2_rs6962824 & 21 & 4465119224232290331515602 \\
\hline KCNN3_rs6426998 & CNTNAP2_rs2972112 & 21 & 4465146224227380515599602 \\
\hline KCNN3_rs6426998 & CNTNAP2_rs7803315 & 20 & 4465119146224227232417602 \\
\hline KCNN3_rs6426998 & NFASC_rs6593917 & 21 & 4465146224232290417515599 \\
\hline KCNN3_rs6426998 & SCN2A_rs17185905 & 20 & 4465119146224227232331515 \\
\hline KCNN3_rs6426998 & ANK3_rs1380452 & 21 & 4465146224227290417515599 \\
\hline KCNN3_rs6426998 & CREB1_rs10932201 & 21 & 4465224232290331417515602 \\
\hline CNTNAP2_rs4493828 & IMPA2_rs3889500 & 20 & 65380388417449469484570574599 \\
\hline CNTNAP2_rs4493828 & SLC12A6_rs8028501 & 20 & 65380388417449469484570574599 \\
\hline CNTNAP2_rs4493828 & TNC_rs1330351 & 21 & 65380388417449469484570574599 \\
\hline CNTNAP2_rs4493828 & TNC_rs2071520 & 21 & 65380388417449469484570574599 \\
\hline CNTNAP2_rs4493828 & ANK3_rs16914571 & 20 & 380388417449469484570574599 \\
\hline CNTNAP2_rs4493828 & AQP4_rs151245 & 21 & 65380388449469484570574599 \\
\hline CNTNAP2_rs4493828 & MBP_rs2282557 & 20 & 65380388417449469484570574599 \\
\hline CNTNAP2_rs4493828 & MBP_rs470330 & 20 & 65380388417449484570574599 \\
\hline CNTNAP2_rs4493828 & MBP_rs470131 & 20 & 65380388417449484570574599 \\
\hline CNTNAP2_rs4493828 & DLG4_rs2586539 & 20 & 65380388417449469570574599 \\
\hline CNTNAP2_rs4493828 & KCNC2_rs1880840 & 20 & 65380388417449469484570574 \\
\hline CNTNAP2_rs4493828 & SLC12A6_rs16958875 & 20 & 65380388417449484570574599 \\
\hline CNTNAP2_rs2972112 & SLC12A6_rs4577050 & 00 & 4465146224292331449498515522570 \\
\hline CNTNAP2_rs2972112 & SLC12A6_rs436552 & 00 & 44146224292331449498515522 \\
\hline CNTNAP2_rs2972112 & PDE4B_rs599381 & 01 & 4483146227232292420498522 \\
\hline CNTNAP2_rs2972112 & KCNC1_rs757511 & 02 & 4465119146331415420449498 \\
\hline CREB1_rs2551645 & P2RX7_rs6489794 & 11 & 44145227290292330420449484533 \\
\hline CREB1_rs2551645 & ANK3_rs9888033 & 10 & 44145227265307330380415436 \\
\hline PPP2R2C_rs6814782 & NRG1_rs4236709 & 00 & 113335415417420436449515533 \\
\hline PPP2R2C_rs6814782 & CAMKK2_rs2686343 & 01 & 44113119290380415515522533 \\
\hline CNTNAP2_rs1024676 & SPTBN4_rs4803342 & 00 & 224265307388415417436533570574 \\
\hline CNTNAP2_rs1024676 & NRG1_rs2466051 & 01 & 44224330388469504522533599 \\
\hline CACNG2_rs738974 & BACE1_rs525493 & 12 & 65290292335388420504533560 \\
\hline CACNG2_rs738974 & BACE1_rs477036 & 12 & 65290292335388420504533560 \\
\hline KCNC3_rs636567 & PPP2R2C_rs10937735 & 11 & 4483119146265290335388522533560 \\
\hline KCNC3_rs636567 & CNTNAP2_rs2373289 & 11 & 83119146335388516522533560 \\
\hline CNTNAP2_rs1730399 & KCNQ2_rs6090403 & 12 & 65113388420449471515516602 \\
\hline PPP2R2C_rs10937735 & SCN2A_rs3769949 & 10 & 44113146227265307417471515522533 \\
\hline BACE1_rs525493 & SCN2A_rs2060199 & 21 & 65290323335388420504533560 \\
\hline CNTN1_rs1596509 & KCNC2_rs1458613 & 01 & 6583145227232330388504533574 \\
\hline MAP2_rs2663652 & KCNN3_rs906280 & 11 & 224232307331388415482484504515602 \\
\hline NCAM1_rs2196456 & CACNG2_rs926543 & 01 & 65145290292330498515516574 \\
\hline SCN5A_rs7430407 & MAP2_rs6733319 & 10 & 44146224265335388417436482 \\
\hline PPP2R2C_rs4386675 & KCNN3_rs883319 & 11 & 83292331388482484497516533574 \\
\hline
\end{tabular}


Table 3. Cont.

\begin{tabular}{llll}
\hline SNP2 & SNP3 & GT $^{\mathbf{a}}$ & Patients $^{\mathbf{b}}$ \\
\hline NFASC_rs16854930 & SCN4B_rs678262 & 10 & 119265330335388436516533570 \\
KCNN3_rs7547552 & NCAM1_rs1807939 & 10 & 6583145265331388484497515516560574599 \\
\hline
\end{tabular}

Cluster 3 contains 41 patients, 49 combinations of 3 SNP genotypes, and 65 SNP genotypes.

a) GT = genotype for SNP2 and SNP3 (0: Normal homozygote.1: Heterozygote. 2: Variant homozygote); b) Dummy ID.

doi:10.1371/journal.pone.0044623.t003

Table 4. Cluster 4. defined by SNP1 $=$ KCNQ3_rs2469515 $=2$.

\begin{tabular}{|c|c|c|c|}
\hline SNP2 & SNP3 & $\mathbf{G T}^{\mathbf{a}}$ & Patients $^{b}$ \\
\hline ANK3_rs12049756 & SCN2A_rs3769949 & 11 & 111268294358360385399444491521538 \\
\hline ANK3_rs12049756 & SCN2A_rs997508 & 11 & 111294354358360385399444491521538 \\
\hline ANK3_rs12049756 & SCN2A_rs12469667 & 11 & 13111294354358360399444491538 \\
\hline ANK3_rs12049756 & ANK3_rs10821702 & 11 & 13111196268294354360385399 \\
\hline ANK3_rs12049756 & AQP4_rs9951307 & 10 & 98111196294360385399521538 \\
\hline SCN5A_rs7430407 & ANK3_rs1010556 & 11 & 1013205672196268328336567 \\
\hline SCN5A_rs7430407 & CNTNAP2_rs4431524 & 11 & 1020565972268328336567 \\
\hline SCN5A_rs7430407 & KCNN3_rs11264250 & 11 & 1320565972268328336567 \\
\hline SPTBN4_rs8107961 & TBR1_rs7564766 & 20 & 0101318196328343358492527599 \\
\hline SPTBN4_rs8107961 & CAMKK2_rs1140886 & 21 & 018196343358360492527538599 \\
\hline ANK3_rs10821677 & ANK3_rs12767186 & 11 & 566298111268294360385399431596 \\
\hline ANK3_rs10821677 & KCNC1_rs757511 & 12 & 6298111294399444521527596 \\
\hline OLIG2_rs762178 & ANK3_rs2393602 & 21 & 02072323328491527538567596 \\
\hline OLIG2_rs762178 & KCNC2_rs11180386 & 20 & 02072323444491492527538 \\
\hline CNTNAP2_rs2462603 & ANK3_rs10994200 & 20 & 206272189268354358538567596 \\
\hline CNTNAP2_rs2462603 & ANK3_rs10761454 & 20 & 206272189268358538567596 \\
\hline PPP2R2C_rs2269920 & TRPM2_rs9974831 & 11 & 02072111323336343354360521562 \\
\hline PPP2R2C_rs2269920 & KCNC3_rs683856 & 11 & 196268294354360369492527562 \\
\hline MAG_rs1034597 & CNTNAP2_rs2972112 & 10 & 101318111294358360385444483538 \\
\hline KCNQ2_rs6089908 & NFASC_rs6677763 & 01 & 294323336343354358492527567596 \\
\hline CNTN1_rs3794247 & NRG1_rs4535704 & 01 & 0106298111294385399491492 \\
\hline ANK3_rs1380452 & TNC_rs1330351 & 01 & 0294328343399444521527567 \\
\hline NRG1_rs2439311 & IMPA2_rs662383 & 11 & 56111328354360431483567599 \\
\hline P2RX7_rs7958311 & BACE1_rs522843 & 11 & 5998111189196294360399431 \\
\hline CNTNAP2_rs4431524 & MBP_rs470826 & 11 & 20565972328354358399527596 \\
\hline DLG4_rs507506 & SPTBN4_rs814501 & 02 & 1318196328360492521538599 \\
\hline IMPA2_rs628419 & KCNQ2_rs6062925 & 12 & 207298268323336358431527 \\
\hline PPP2R2C_rs3796403 & IMPA2_rs3786305 & 11 & 05998111196336358431483 \\
\hline CNTNAP1_rs2271029 & NRCAM_rs6958498 & 11 & 1013196354399492527538562 \\
\hline KCNQ2_rs884851 & CNTNAP1_rs9897724 & 10 & 056294328369385431562596 \\
\hline NRG1_rs3924999 & ANK3_rs10761482 & 11 & 10135998294360399431521 \\
\hline CREB1_rs2551921 & MBP_rs9676113 & 11 & 182072369399483527567599 \\
\hline
\end{tabular}

Cluster 4 contains 37 patients, 32 combinations of 3 SNP genotypes, and 53 SNP genotypes.

a) GT = genotype for SNP2 and SNP3 (0: Normal homozygote.1: Heterozygote. 2: Variant homozygote); b) Dummy ID.

doi:10.1371/journal.pone.0044623.t004 


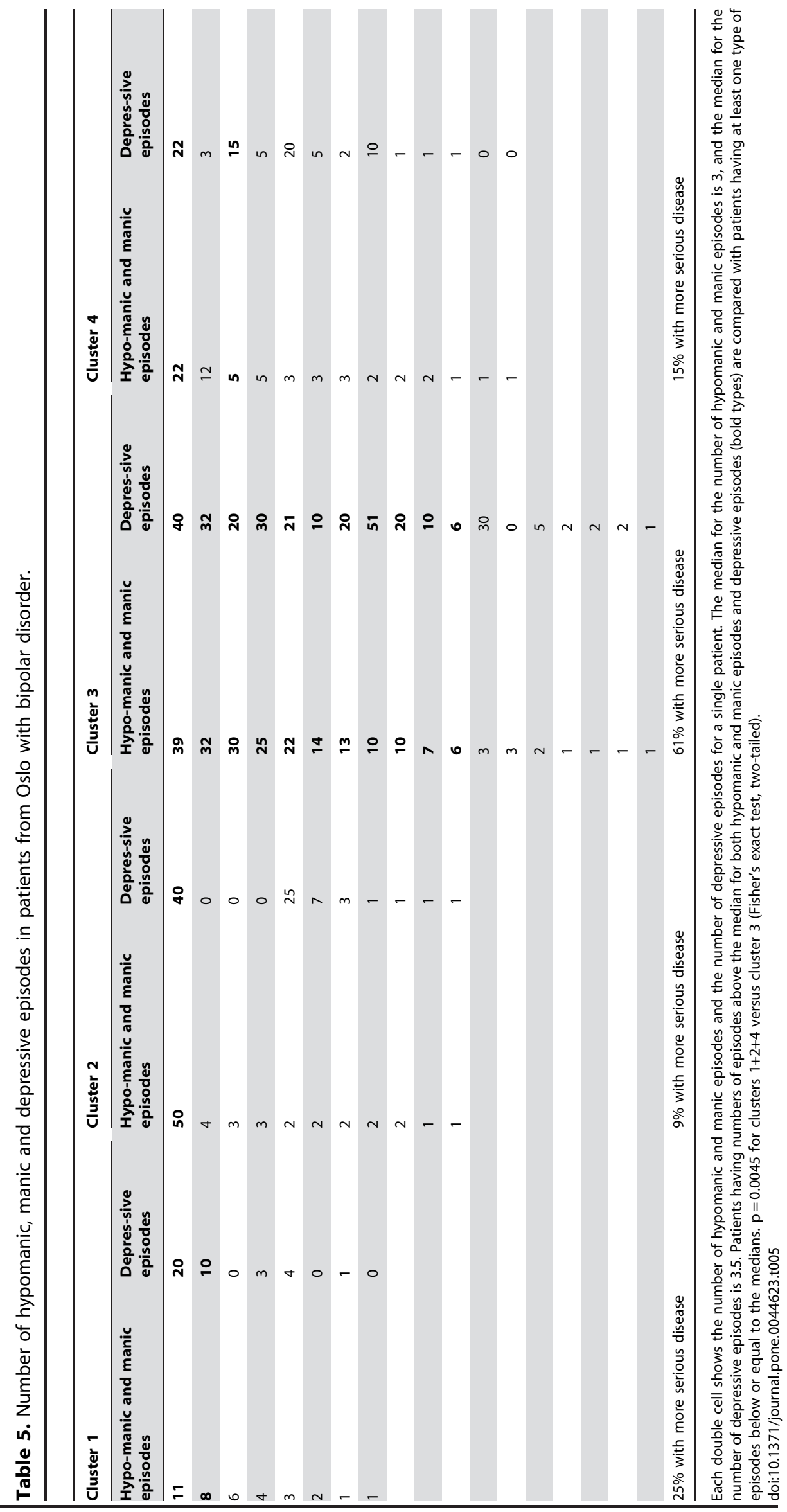


Table 6. Number of hospital admissions for patients with bipolar disorder in Copenhagen.

\begin{tabular}{|c|c|c|c|}
\hline Cluster 1 & Cluster 2 & Cluster 3 & Cluster 4 \\
\hline 38 & 38 & 14 & 70 \\
\hline 25 & 32 & 12 & 41 \\
\hline 13 & 22 & 8 & 40 \\
\hline 7 & 17 & 4 & 20 \\
\hline 7 & 12 & 3 & 19 \\
\hline 6 & 9 & & 11 \\
\hline 5 & 5 & & 7 \\
\hline 5 & 5 & & 7 \\
\hline 3 & 4 & & 6 \\
\hline 3 & 4 & & \\
\hline 2 & 3 & & \\
\hline 1 & 3 & & \\
\hline 1 & 3 & & \\
\hline \multirow[t]{5}{*}{0} & 2 & & \\
\hline & 2 & & \\
\hline & 2 & & \\
\hline & 0 & & \\
\hline & 0 & & \\
\hline
\end{tabular}

Table 7. Alcohol dependence (1) or non-dependence (0) in patients from Copenhagen with bipolar disorder.

\begin{tabular}{llll}
\hline & & & \\
\hline Cluster 1 & Cluster 2 & Cluster 3 & Cluster 4 \\
\hline 0 & 0 & 0 & 0 \\
0 & 0 & 1 & 0 \\
0 & 0 & 1 & 0 \\
0 & 0 & 1 & 1 \\
0 & 0 & & 1 \\
0 & 0 & & 1 \\
0 & 0 & & \\
0 & 0 & & \\
0 & 0 & & \\
0 & 0 & & \\
1 & 0 & & \\
1 & 1 & & \\
1 & 1 & & \\
\hline Each box represents one patient. & & \\
doi:10.1371/journal.pone.0044623.t007 & & \\
\hline
\end{tabular}

\section{Discussion}

The four clusters of combinations of SNP-genotypes were statistically significantly associated with bipolar disorder; whereas biological or clinical significance of the clusters was not apparent, apart from the original selection of genes related to signal transduction [3]. These genes are shown in Table 8 . The relatively little overlap between the patients in the clusters led to an analysis of available clinical data from the psychiatric departments that had recruited the patients from three locations in Scandinavia. The division of patients according to locations, clusters and availability of clinical data led to the small groups of patients shown in Tables 5-7.

Using numbers of hypomanic, manic and depressive episodes higher than the median for these episodes as an indication of severity of disease, it was found that the number of patients with more severe disease was higher in one cluster compared with the three other clusters (Table 5). This result suggests that it may be possible to connect combinations of genetic data to clinical data. The figures in Table 6 and 7 may led to similar suggestions, but although significant differences may be found between the distributions in these tables, the statistical power is low and no significant results may remain after correction for multiple testing.

Due to the relatively low number of patients as well as of clinical data, no strong conclusions can be drawn from this study. However, the results in Table 5 indicated that some genetic subgroups may be more affected by their illness than other subgroups, hereby justifying further work with combinations of genetic data as a method to connect genetic and clinical data. Hopefully, other studies with more patients, more genetic data and more clinical data will try to look at combinations of their data.

\section{Materials and Methods}

The patient sample, genes, SNP selection and genotyping, statistics and data processing regarding Table 1, 2, 3, 4 were described previously [3]. The Norwegian Scientific-Ethical Committees, the Norwegian Data Protection Agency, the Danish Scientific Committees, and the Danish Data Protection Agency approved the study. All patients gave written informed consent prior to inclusion in the project. The data in Table 5 were analyzed statistically with Fisher's exact test, two-tailed. In Tables 5, 6, 7 each box represents one patient. The numbers of hypomanic, manic, and depressive episodes in Norwegian patients were obtained by SCID [8]. The numbers of admissions in Copenhagen were obtained from patient records. Patients from Copenhagen were diagnosed with alcohol dependence when the patient was, or had been, treated in an alcohol clinic.

\section{Author Contributions}

Conceived and designed the experiments: EM PK. Analyzed the data: EM PK GLM. Contributed reagents/materials/analysis tools: HD BB IM OAA SD OM TH. Wrote the paper: EM PK. 
Table 8. Selected genes and function [3].

\begin{tabular}{|c|c|c|}
\hline Gene & Location & Name and/or Function \\
\hline ANK3 & $10 q 21$ & Role for structure and function of nodes of Ranvier \\
\hline$A Q P 4$ & $18 q 11.2-q 12.1$ & Regulator of vasopression secretion \\
\hline ATP1A2 & $1 q 21-q 23$ & $\mathrm{Na}+/ \mathrm{K}+$ ATPase alpha-2 subunit \\
\hline ATP1A3 & $19 q 13.31$ & $\mathrm{Na}+/ \mathrm{K}+$ ATPase alpha-3 subunit \\
\hline AVPR1B & $1 q 32$ & Arginine vasopressin receptor $1 \mathrm{~B}$ \\
\hline BACE1 & $11 q 23.2-q 23.3$ & Regulation of the voltage dependent Na-channels. \\
\hline$B D N F$ & $11 \mathrm{p} 13$ & Involved in neuroplasticity and stress response \\
\hline CACNG2 & $22 q 13.1$ & Neuronal calcium channel gamma subunit, stabilize the channel in an inactive state \\
\hline CAMKK2 & $12 q 24.2$ & Involved in activation of CREB1 \\
\hline CLDN11 & $3 q 26.2-q 26.3$ & Role in myelinisation \\
\hline CNTN1 & $12 q 11-q 12$ & Cell adhesion molecule \\
\hline CNTN2 & $1 \mathrm{q} 32.1$ & Cell adhesion molecule \\
\hline CNTNAP1 & $17 q 21$ & Contactin-associated protein, may be the signaling subunit of contactin \\
\hline CNTNAP2 & $7 q 35-q 36$ & Cluster voltage-gated potassium channels, localized at the juxtaparanodes \\
\hline CREB1 & $2 q 34$ & Transcription factor \\
\hline DLG4 & $17 \mathrm{p} 13.1$ & Neuronal development, recruited into potassium channel clusters \\
\hline ERBB4 & $2 q 33.3-q 34$ & Neuregulin-1 receptor, involved in mitogenesis and differentiation \\
\hline GSK3B & $3 q 13.3$ & Neuronal cell development (Related to lithium respons) \\
\hline IMPA2 & $18 p 11.2$ & Inositol monophosphatase (Related to lithium respons) \\
\hline KCNA1 & $12 \mathrm{p} 13.32$ & Voltage-gated delayed potassium channel \\
\hline KCNA2 & $1 \mathrm{p} 13$ & Voltage-gated delayed potassium channel, delayed rectifier class \\
\hline KCNC1 & $11 \mathrm{p} 15$ & Mediates the voltage-dependent potassium ion permeability of excitable membranes \\
\hline KCNC2 & $12 q 14.1$ & Mediates the voltage-dependent potassium ion permeability of excitable membranes \\
\hline KCNC3 & $19 q 13.3-q 13.4$ & Mediates the voltage-dependent potassium ion permeability of excitable membranes \\
\hline KCNN3 & $1 \mathrm{q} 21.3$ & Potassium conductance Ca-activited channel, regulate neuronal excitability \\
\hline KCNQ2 & $20 q 13.3$ & Voltage-gated potassium channel plays a role in the regulation of neuronal excitability \\
\hline KCNQ3 & $8 q 24$ & Voltage-gated potassium channel plays a role in the regulation of neuronal excitability \\
\hline MAG & $19 q 13.1$ & Central role i myelinisation, involved in myelin-neuron cell-cell interactions \\
\hline MAP2 & $2 q 34-q 35$ & Microtubule-associated protein, involved in neurogenesis \\
\hline MBP & $18 \mathrm{q} 23$ & Major constituent of the myelin sheath of oligodendrocytes in the nervous system \\
\hline MCHR1 & $22 q 13.2$ & Inhibit cAMP accumulation stimulate intracellular Ca-flux \\
\hline MCTP2 & $15 q 26.2$ & Intercellular signal transduction \\
\hline MOG & $6 \mathrm{p} 22.1$ & Involved in completion and maintenance of the myelin sheath and in cell-cell communication \\
\hline NCAM1 & $11 \mathrm{q} 23.1$ & Neural cell adhesion molecule 1 \\
\hline NFASC & $1 \mathrm{q} 32.1$ & Cell adhesion; organization of the axon initial segment (AIS) and nodes of Ranvier \\
\hline NRCAM & $7 q 31.1-q 31.2$ & Ankyrin-binding protein is involved in neuron-neuron adhesion \\
\hline NRG1 & $8 p 12$ & Associated with ERBB receptors \\
\hline NTRK1 & $1 q 21-q 22$ & Neurotrophic tyrosine kinase, receptor, type 1 \\
\hline OLIG2 & $21 \mathrm{q} 22.11$ & Oligodendrocyte lineage transcription factor 2 \\
\hline$P 2 R X 7$ & $12 q 24$ & Ligand-gated ion channel \\
\hline PDE4B & $1 \mathrm{p} 31$ & Phosphodiesterase 4B, cAMP-specific \\
\hline$P P P 2 R 2 C$ & $4 p 16.1$ & Protein phosphatase 2 , regulatory subunit $B$, gamma isoform \\
\hline SCN1B & $19 q 13.1$ & Sodium channel beta subunit, propagation of nerveimpulses, binding to contactin \\
\hline$S C N 2 A$ & $2 q 23-q 24$ & Sodium channel alpha subunit, generation and propagation of action potentials in neurons \\
\hline$S C N 2 B$ & $11 \mathrm{q} 23$ & Sodium channel, voltage-gated, type II, beta \\
\hline$S C N 4 B$ & $11 \mathrm{q} 23.3$ & Sodium channel, voltage-gated, type IV, beta \\
\hline SCN5A & $3 p 21$ & Sodium channel, voltage-gated, type $V$, alpha subunit \\
\hline SCN8A & $12 q 13$ & Sodium channel, voltage gated, type VIII, alpha subunit, associated with ANK3 \\
\hline SLC12A6 & $15 q 13-q 15$ & Electroneutral potassium-chloride cotransporter 3 \\
\hline
\end{tabular}


Table 8. Cont.

\begin{tabular}{lll}
\hline Gene & Location & Name and/or Function \\
\hline SPTBN4 & $19 q 13.13$ & Involved in location of specific membrane proteins in polarized regions of neurons \\
TBR1 & $2 \mathrm{q} 24$ & Transcription factor, critical for early cortical development \\
TNC & $9 \mathrm{q} 33$ & Regulation of Na channels. Interaction with CNTN1 \\
TNR & $1 \mathrm{q} 24$ & Extracellular matix protein expressed primarily in the central nervous system \\
TRPM2 & $21 \mathrm{q} 22.3$ & Transient receptor potential cation channel, subfamily M, member 2 \\
YWHAH & $22 \mathrm{q} 12.3$ & Tyrosine 3-monooxygenase/tryptophan 5-monooxygenase activation protein, eta polypeptide
\end{tabular}

doi:10.1371/journal.pone.0044623.t008

\section{References}

1. Cooper GM, Shendure J (2011) Needles in stacks of needles: finding diseasecausal variants in a wealth of genomic data. Nat Rev Genet 12: 628-640.

2. Abo R, Knight S, Thomas A, Camp NJ (2011) Automated construction and testing of multi-locus gene-gene associations. Bioinformatics 27: 134-136.

3. Koefoed P, Andreassen OA, Bennike B, Dam H, Djurovic S, et al. (2011) Combinations of SNPs Related to Signal Transduction in Bipolar Disorder. PLoS ONE 6: e23812.

4. Askland K, Read C, Moore J (2009) Pathways-based analyses of whole-genome association study data in bipolar disorder reveal genes mediating ion channel activity and synaptic neurotransmission. Hum Genet 125: 63-79.
5. El-Mallakh RS, Huff MO (2001) Mood stabilizers and ion regulation. Harvard Rev Psychiatry 9: 23-32.

6. Mellerup E, Kristensen F (2004) Mania as a dysfunction of reentry: application of Edelman's and Tononi's hypothesis for consciousness in relation to a psychiatric disorder. Med Hypotheses 63: 464-466.

7. Mellerup E, Møller GL, Koefoed P (2012) Genetics of complex disorders. Variation on a theme. Med Hypotheses 78: 732-734.

8. Williams JB, Gibbon M, First MB, Spitzer RL, Davies M, et al. (1992) The Structured Clinical Interview for DSM-III-R (SCID). II. Multisite test-retest reliability. Arch Gen Psychiatry 49: 630-636. 